\title{
Theory and computation of scalar and tensor polarizabilities and hyperpolarizabilities and of LoSurdo-Stark shifts in polyelectronic atoms
}

\author{
Spyros I. Themelis and Cleanthes A. Nicolaides \\ Theoretical and Physical Chemistry Institute, National Hellenic Research Foundation, \\ 48 Vassileos Constantinou Avenue, 16635 Athens, Greece
}

(Received 28 February 1992)

\begin{abstract}
By applying an all-order, complex eigenvalue polyelectronic theory, applicable to ground or excited states, we have obtained electric field-dependent energy shifts for the $\mathrm{Li} 1 s^{2} 2 s^{2} S$ and $1 s^{2} 2 p^{2} P^{\circ}$ states for field strengths in the range 0.0-0.02 a.u. These results are then used in conjunction with the appropriate expressions for the scalar and tensor polarizabilities $\left(\alpha_{0}, \alpha_{2}\right)$ and hyperpolarizabilities $\left(\gamma_{0}, \gamma_{2}\right)$ of $P$ states, to obtain these quantities for the $\mathrm{Li} 1 s^{2} 2 p^{2} P^{\circ}$ state. Whereas the linear anisotropy is negligible $\left(\alpha_{0}=20.11 \AA^{3}, \alpha_{2}=0.019 \AA^{3}\right)$, the nonlinear one is huge $\left(\gamma_{0}=2.42 \times 10^{5} \AA^{6}, \gamma_{2}=-1.53\right.$ $\times 10^{5} \AA^{6}$ ), a fact implying observable anisotropic effects in strong laser fields. The difference between the $\alpha_{0}$ of the ${ }^{2} P^{\circ}$ and ${ }^{2} S$ states is $\delta \alpha_{0}=4.93 \AA^{3}$, in fairly good agreement with the recent measurement $\left(\delta \alpha_{0}=5.51 \AA^{3}\right)$ of Hunter et al. [Phys. Rev. A $446140(1991)$ ].
\end{abstract}

PACS number(s): $32.60 .+\mathrm{i}, 32.70 . \mathrm{Jz}$

\section{INTRODUCTION}

In a recent paper, Hunter et al. [1] reported a "precise measurement of the Stark shift of the lithium $D_{1}$ line" [4.62 $\left.\mathrm{kHz} /(\mathrm{kV} / \mathrm{cm})^{2}\right]$, from which they obtained the difference between the scalar polarizabilities of the $\mathrm{Li}$ $1 s^{2} 2 s^{2} S$ and $1 s^{2} 2 p^{2} P^{\circ}$ states, $\delta \alpha_{0}=5.51 \AA^{3}$. Combining this number with the measured value [2] of $\alpha_{0}\left({ }^{2} S\right)=24.3$ $\AA^{3}$, they determined the scalar polarizability of the excited state, $\alpha_{0}\left({ }^{2} P^{\circ}\right)=18.8 \AA^{3}$. An increased accuracy by one digit is obtained if the accurate theoretical value [3] for the $\alpha_{0}\left({ }^{2} S\right)$ is used $\left(24.27 \AA^{3}\right)$. In this case, the experimental result gives $\alpha_{0}\left({ }^{2} P^{\circ}\right)=18.76 \AA^{3}$.

TABLE I. Scalar and tensor polarizabilities $\left(\alpha_{0}, \alpha_{2}\right.$ in $\AA^{3}, 1$ a.u. $\left.=0.1482 \AA^{3}\right)$ and hyperpolarizabilities $\left(\gamma_{0}, \gamma_{2}\right.$ in $\AA^{6}, 1$ a.u. $=0.02196 \AA^{6}$ ) [Eqs. (3) and (7)] for the Li $1 s^{2} 2 p^{2} P^{\circ}$ state and difference $(\delta \alpha)$ between the scalar polarizabilities of the $\mathrm{Li}$ $1 s^{2} 2 s^{2} S$ and the ${ }^{2} P^{\circ}$ states. The measurement of Hunter $e t$ al. [1] gave $\delta \alpha_{0}$ directly. The experimental $\alpha_{0}\left({ }^{2} P^{\circ}\right)$ is obtained by combining the accurate theoretical $\alpha_{0}\left({ }^{2} S\right)\left(24.27 \AA^{3}\right)$ [3] with the measured $\delta \alpha_{0}$. The results of Refs. [4,5] were computed using perturbation theory and semiempirically obtained orbitals. The calculations of Ref. [4] were done for the $J=\frac{3}{2}$ level. Our results are obtained from the solution of the CESE of Eqs. (1), (2), and (10) and from the use of Eqs. (3)-(7). Note that whereas $\alpha_{2}$ is negligible compared to $\alpha_{0}$, this is not the case for $\gamma_{2}$, whose sign is, in fact, negative.

\begin{tabular}{lcccc}
\hline \hline & Experiment & \multicolumn{3}{c}{ Theory } \\
\cline { 3 - 5 } & {$[1]$} & [4] & [5] & This work \\
\hline$\alpha_{0}\left({ }^{2} P^{\circ}\right)\left(\AA^{3}\right)$ & 18.76 & 23.8 & 21.5 & 20.11 \\
$\alpha_{2}\left({ }^{2} P^{\circ}\right)\left(\AA^{3}\right)$ & & -5.9 & & 0.019 \\
$\delta \alpha_{0}\left({ }^{2} S-{ }^{2} P^{\circ}\right)\left(\AA^{3}\right)$ & 5.512 & & 2.8 & 4.93 \\
$\gamma_{0}\left({ }^{2} P^{\circ}\right)\left(\AA^{6}\right)$ & & & & $2.42 \times 10^{5}$ \\
$\gamma_{2}\left({ }^{2} P^{\circ}\right)\left(\AA^{6}\right)$ & & & & $-1.53 \times 10^{5}$ \\
\hline \hline
\end{tabular}

The available theoretical values for the scalar and tensor polarizabilities of the $\mathrm{Li}^{2} P^{\circ}$ state $[4,5]$ were obtained from expressions of perturbation theory with one-electron radials from the semiempirical Bates-Damgaard method. Table I shows that there are discrepancies. Also, the tensor polarizability, $\alpha_{2}$, has been predicted to have a significant magnitude and a negative sign [4].

In this paper we present a theoretical result for $\delta \alpha_{0}$ of the $\mathrm{Li}, D_{1}$ line, together with a number of other results pertaining to the computation of polarization properties of excited states of polyelectronic neutral atoms. These are obtained by applying a recently developed nonperturbative polyelectronic theory of the field-induced energy shift, $\Delta(F)[6-9]$. The theory permits the computation of $\Delta(F)$ to all orders and therefore is suitable for treating the strong-field regime. At the same time, by fitting $\Delta(F)$, as $F \rightarrow 0$, to a Taylor series, scalar as well as tensor polarizabilities and hyperpolarizabilities can be computed. Our value for $\alpha_{2}$ is much smaller than that of Schmieder, Lurio, and Happer [4] (see Table I). On the other hand, the scalar, $\left(\gamma_{0}\right)$, and tensor, $\left(\gamma_{2}\right)$, hyperpolarizabilities are of the same magnitude.

\section{THEORY}

Our theory of field-induced energy shifts and of linear $(\alpha)$ and nonlinear $(\gamma)$ polarizabilities has been formulated as a problem of obtaining solutions to a complex eigenvalue Schrödinger equation (CESE) defining resonance states $\Psi[6-9]$ :

$$
\begin{aligned}
& \left(H-z_{0}\right) \Psi=0, \\
& z_{0}=E_{0}+\Delta(F)-\frac{1}{2} i \Gamma(F) .
\end{aligned}
$$

The Hamiltonian $H$ includes the external perturbation of strength $F$, while $E_{0}$ corresponds to the unperturbed energy of the state under consideration, whose square integrable wave function is obtained according to the state- 
specific theory of the electronic structures. The energy shift $\Delta(F)$ and width $\Gamma(F)$ are functions of the field strength (and, for ac fields, of the frequency).

The computation is nonperturbative and produces the energy shifts to all orders. Therefore it can handle, formally as well as practically, the case of strong fields. Furthermore, having been formulated around statespecific Hartree-Fock (HF) or MCHF zeroth-order functions to which correlation corrections are added, it is applicable efficiently to ground or excited states, regardless of whether the reference function is single determinantal or multiconfigurational.

Having obtained $\Delta(F)$ (Sec. III) for each independent azimuthal quantum number $M$, the various polarizabilities computed here were obtained from

$$
\Delta(F)=-\frac{1}{2 !} \alpha_{n L M} F^{2}-\frac{1}{4 !} \gamma_{n L M} F^{4}-\cdots,
$$

where the coefficients $\alpha$ and $\gamma$ are adapted to the corresponding expressions of Davydkin and Ovsiannikov [10]. Thus,

$$
\alpha_{n L M}=\sum_{k=0}^{1}(-1)^{L+M-2 k} \sqrt{2 L+1}\left(\begin{array}{ccc}
L & 2 k & L \\
M & 0 & -M
\end{array}\right)\left[\frac{(2 L+2)_{2 k}}{(2 L+1-2 k)_{2 k}}\right]^{1 / 2} a_{2 k},
$$

where the term in large parentheses is the $3 j$ symbol and $(b)_{p}$ is the Pochhammer symbol. Using standard angular momentum theory, $\alpha_{n L M}$ is rewritten as

$$
\alpha_{n L M}=\alpha_{0}+\alpha_{2} \frac{3 M^{2}-L(L+1)}{L(2 L-1)},
$$

where $\alpha_{0}$ is the scalar polarizability and $\alpha_{2}$ is the tensor polarizability. Similarly, the nonlinear polarizability, $\gamma_{n L M}$, can be written as [10]

$$
\gamma_{n L M}=\sum_{k=0}^{2}(-1)^{L+M-2 k} \sqrt{2 L+1}\left(\begin{array}{ccc}
L & 2 k & L \\
M & 0 & -M
\end{array}\right)\left[\frac{(2 L+2)_{2 k}}{(2 L+1-2 k)_{2 k}}\right]^{1 / 2} \gamma_{2 k}
$$

Since for $k>2 \gamma_{2 k}$ is zero, for the ${ }^{2} P^{\circ}$ state we obtain

$$
\gamma_{n L M}=\gamma_{0}+\gamma_{2} \frac{3 M^{2}-L(L+1)}{L(2 L-1)} \text {. }
$$

The quantities in Eqs. (5) and (7) are obtained after a polynomial fit of $\Delta(F)$ as $F \rightarrow 0$ is made for $M_{L}=0$ and $M_{L}=+1$. These values are $\alpha\left(M_{L}=0\right)=135.44$ a.u., $\alpha\left(M_{L}= \pm 1\right)=135.82$ a.u., $\gamma\left(M_{L}=0\right)=2.509 \times 10^{7}$ a.u. $\gamma\left(M_{L}= \pm 1\right)=4.199 \times 10^{6}$ a.u. The corresponding values for $\alpha_{0}, \gamma_{0}, \alpha_{2}$, and $\gamma_{2}$ are given in Table I.

\section{THE COMPUTATION OF $\Delta(F)$}

The form of the trial functions for the dressed states " $1 s^{2} 2 s^{2} S$ " and " $1 s^{2} 2 p^{2} P^{\circ} "$ was taken from the statespecific theory of decaying states (e.g. $[6-9,11]$ ), where emphasis is given to the proper choice of separately optimized function spaces, according to the spectrum under consideration and the physics of decay. This form is

$$
\Psi=\sum_{i} a_{i} \Phi_{i}+\sum_{j} b_{j} X_{j}
$$

Since we deal with a neutral system, the external perturbation can couple discrete states, $\Phi_{i}$, of different symmetry and parity. The set of $\left\{\Phi_{i}\right\}$ will be taken to define a space $Q$ with symmetries $S, P, D$, and $F$. The corresponding configurational assignments are $1 s^{2} n l$, where $n=2,3$, 4 and $l=0,1,2,3$. The wave functions representing these states were computed at the state-specific HF level. Such functions account for $1 / r_{i j}$-induced valence spin-orbital polarization (e.g., $2 p \rightarrow 3 p$ ) as well as for field-induced angular momentum mixing. However, they do not account for $1 / r_{i j}$-induced $\left(1 s^{2}\right)$ core polarization and inter- shell correlation effects whose overall effect is known to reduce somewhat the magnitude of alkali polarizabilities (Ref. [3] and previous work). On the other hand, since the polarizability of $\mathrm{Li}^{+} 1 s^{2} S$ is extremely small, $\left(\alpha=0.028 \AA^{3}[12]\right)$, field-induced core single excitations are negligible and were not included in $\Psi$ [13]. In view of the above, and given that, as regards the measured quantity $\delta \alpha$, certain core orbital correlation contributions to the polarization of the $\mathrm{Li}^{2} S$ and ${ }^{2} P^{\circ}$ should cancel, the extra effort involved in using correlated functions for each $\Phi_{i}$ was considered uneconomical and was not pursued.

The final-state terms were taken as

$$
X_{j}=A\left[\mathrm{Li}^{+}\left(1 s^{2}\right) \otimes g_{j}\right],
$$

where $A$ is the antisymmetrizer and $g_{j}$ is the Gamow orbital which is expanded in terms of Slater-type orbitals (STOs) with complex coordinate $\rho^{*}=r e^{-i \theta}[6-9]$ representing the high-lying Rydberg and continuum orbital function space.

Construction of the trial function as in Eq. (8) allows one to focus on the specifics of the bases and to keep the computation tractable regardless of the number of electrons of the atomic or molecular system under examination. Thus, the compactness of the state-specific expansion of the first term permits the inclusion in the second term of a large number of angular momenta in the expansion of the Gamow orbital, a prerequisite for wellstabilized and reliable results for problems requiring the solution of Eq. (1) with external fields. The size of this expansion depends on the strength of the field. In the present work, for large fields we used $9 s, 9 p, 8 d, 7 f, 6 g$, $6 h, 5 i, 5 k, 4 l$, and $4 m$ STOs, which were kept orthogonal to each other but not to the bound HF orbitals. The nonlinear parameter of these STOs was optimized (see below) 


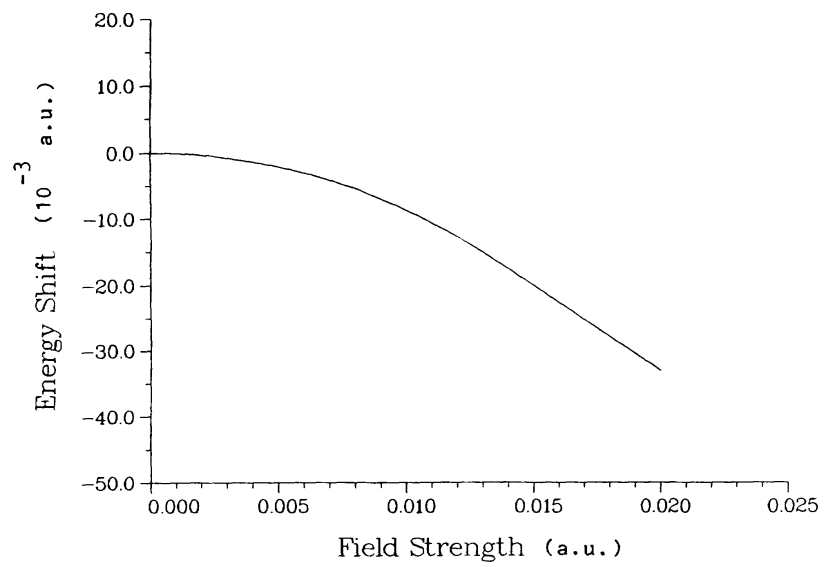

FIG. 1. Field-induced energy shift from the zero-field energy of the Li $1 s^{2} 2 s^{2} S$ state.

to the value $\beta=0.7$.

Let $Q$ represent the function space of the $\Phi_{i}$ and $P$ the function space defined by $X_{j}$. Then, the complex symmetric non-Hermitian Hamiltonian matrix

$$
\mathbf{H}=\left(\begin{array}{ll}
H_{Q Q} & H_{Q P} \\
H_{P Q} & H_{P P}
\end{array}\right)
$$

was constructed and diagonalized repeatedly as a function of the nonlinear parameter in the STOs and of the angle $\theta$, until stable results were obtained for the root whose overlap with the HF $1 s^{2} 2 s^{2} S$ or $1 s^{2} 2 p^{2} P^{\circ}$ function was maximum.

\section{RESULTS}

Our results are presented in Table I for the polarizabilities and hyperpolarizabilities of $\mathrm{Li}^{2} P^{\circ}$ and Figs. 1-3 and Table II for the energy shifts of the $\mathrm{Li}^{2} S$ and ${ }^{2} P^{\circ}$ states, which, for small values of the field strength, feature the expected quadratic character. As the strength increases, there is a range where the dependence becomes approximately linear, suggesting the dominance of two-state cou-

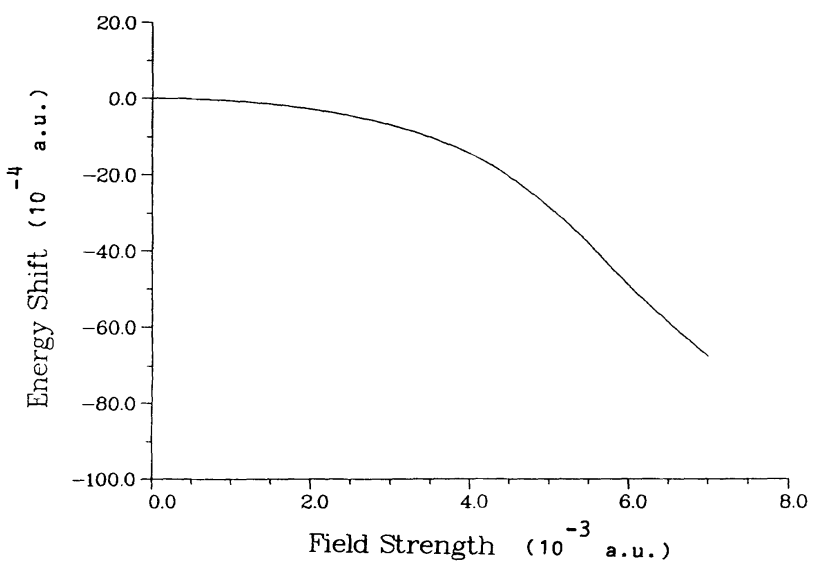

FIG. 2. Field-induced energy shift from the zero-field energy of the Li $1 s^{2} 2 p^{2} P^{\circ}\left(M_{L}=0\right)$ state.

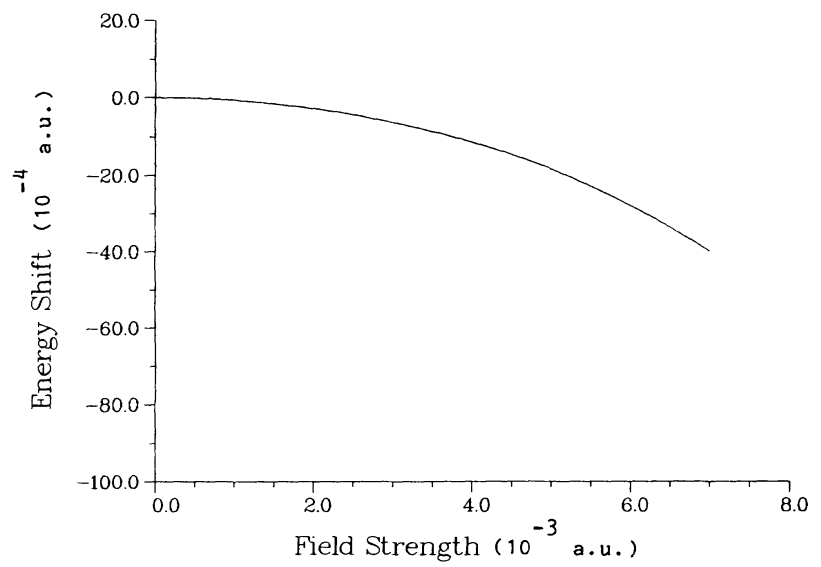

FIG. 3. Field-induced energy shift from the zero-field energy of the $\mathrm{Li} 1 s^{2} 2 p^{2} P^{\circ}\left(M_{L}= \pm 1\right)$ state.

pling (e.g., [14]). For still stronger fields, the collective contribution of many terms leads to a complicated $M_{L}$ and state-specific dependence.

Table II shows that the energies of the $\mathrm{Li}^{2} S$ and ${ }^{2} \mathrm{P}^{\circ}$ states are shifted by almost the same amount (order of magnitude) by electric fields of strength $F=1 \times 10^{-4}$ $25 \times 10^{-4}$ a.u.

For the ground state, $\mathrm{Li} 1 s^{2} 2 s^{2} S$, the computed polarizability is $\alpha_{0}=25.09 \AA^{3}$. The $3 \%$ deviation from the ac-

TABLE II. Field-induced energy shift from the zero-field energy for $F=1 \times 10^{-4}-2.5 \times 10^{-3}$ a.u.

\begin{tabular}{cccc}
\hline \hline $\begin{array}{c}F \\
\text { (a.u.) }\end{array}$ & $\begin{array}{c}\Delta\left({ }^{2} S\right) \\
(\mathrm{eV})\end{array}$ & $\begin{array}{c}\Delta\left({ }^{2} P^{\circ}, M_{L}=0\right) \\
(\mathrm{eV})\end{array}$ & $\begin{array}{c}-\Delta\left({ }^{2} P^{\circ}, M_{L}= \pm 1\right) \\
(\mathrm{eV})\end{array}$ \\
\hline 0.0001 & $2.29 \times 10^{-5}$ & $1.85 \times 10^{-5}$ & $1.85 \times 10^{-5}$ \\
0.0002 & $9.20 \times 10^{-5}$ & $7.37 \times 10^{-5}$ & $7.39 \times 10^{-5}$ \\
0.0003 & $2.07 \times 10^{-4}$ & $1.66 \times 10^{-4}$ & $1.66 \times 10^{-4}$ \\
0.0004 & $3.68 \times 10^{-4}$ & $2.96 \times 10^{-4}$ & $2.96 \times 10^{-4}$ \\
0.0005 & $5.75 \times 10^{-4}$ & $4.63 \times 10^{-4}$ & $4.62 \times 10^{-4}$ \\
0.0006 & $8.28 \times 10^{-4}$ & $6.67 \times 10^{-4}$ & $6.66 \times 10^{-4}$ \\
0.0007 & $1.13 \times 10^{-3}$ & $9.10 \times 10^{-4}$ & $9.07 \times 10^{-4}$ \\
0.0008 & $1.47 \times 10^{-3}$ & $1.19 \times 10^{-3}$ & $1.18 \times 10^{-3}$ \\
0.0009 & $1.86 \times 10^{-3}$ & $1.51 \times 10^{-3}$ & $1.50 \times 10^{-3}$ \\
0.0010 & $2.30 \times 10^{-3}$ & $1.87 \times 10^{-3}$ & $1.85 \times 10^{-3}$ \\
0.0011 & $2.78 \times 10^{-3}$ & $2.27 \times 10^{-3}$ & $2.24 \times 10^{-3}$ \\
0.0012 & $3.31 \times 10^{-3}$ & $2.71 \times 10^{-3}$ & $2.67 \times 10^{-3}$ \\
0.0013 & $3.89 \times 10^{-3}$ & $3.20 \times 10^{-3}$ & $3.14 \times 10^{-3}$ \\
0.0014 & $4.51 \times 10^{-3}$ & $3.72 \times 10^{-3}$ & $3.64 \times 10^{-3}$ \\
0.0015 & $5.17 \times 10^{-3}$ & $4.30 \times 10^{-3}$ & $4.18 \times 10^{-3}$ \\
0.0016 & $5.89 \times 10^{-3}$ & $4.91 \times 10^{-3}$ & $4.76 \times 10^{-3}$ \\
0.0017 & $6.65 \times 10^{-3}$ & $5.57 \times 10^{-3}$ & $5.38 \times 10^{-3}$ \\
0.0018 & $7.45 \times 10^{-3}$ & $6.29 \times 10^{-3}$ & $6.04 \times 10^{-3}$ \\
0.0019 & $8.30 \times 10^{-3}$ & $7.05 \times 10^{-3}$ & $6.74 \times 10^{-3}$ \\
0.0020 & $9.20 \times 10^{-3}$ & $7.86 \times 10^{-3}$ & $7.47 \times 10^{-3}$ \\
0.0021 & $1.01 \times 10^{-2}$ & $8.72 \times 10^{-3}$ & $8.25 \times 10^{-3}$ \\
0.0022 & $1.11 \times 10^{-2}$ & $9.64 \times 10^{-3}$ & $9.06 \times 10^{-3}$ \\
0.0023 & $1.22 \times 10^{-2}$ & $1.06 \times 10^{-2}$ & $9.92 \times 10^{-3}$ \\
0.0024 & $1.32 \times 10^{-2}$ & $1.17 \times 10^{-2}$ & $1.08 \times 10^{-2}$ \\
0.0025 & $1.44 \times 10^{-2}$ & $1.28 \times 10^{-2}$ & $1.18 \times 10^{-2}$ \\
\hline \hline
\end{tabular}


curate value [3] is due to polarization and pair correlation effects involving the $1 s 2 s$ and $1 s^{2}$ electrons. When $\alpha_{0}$ of the ${ }^{2} P$ state (Table I) $\left(20.11 \AA^{3}\right)$ is subtracted, we obtain $\delta \alpha_{0}=4.93 \AA^{3}$ in fairly good agreement with measurement $\left(\delta \alpha_{0}=5.51 \AA^{3}\right)$.

Finally, the tensor hyperpolarizability $\left(\gamma_{2}\right)$, computed $a b$ initio for an $L \neq 0$ polyelectronic state for the first time, shows a very interesting difference from $\alpha_{2}$ as regards magnitude and sign relative to the corresponding scalar quantities $\left(\gamma_{2} / \gamma_{0} \approx-1\right.$ where $\left.\alpha_{2} / \alpha_{0} \approx 0\right)$. Assuming a smooth frequency dependence at least for small values of $\omega$, this result implies that when the $\mathrm{Li}^{2} P^{\circ}$ state is exposed to a strong laser field, the anisotropy between the independent susceptibility components will be large.

\section{CONCLUSION}

The use of Eqs. (1)-(10) allows the computation of scalar and tensor polarizabilities and hyperpolarizabilities for ground or excited states of $L \neq 0$ of polyelectronic neutral atoms as well as field-induced energy shifts for strong fields, by solving the complex eigenvalue Schrödinger equation in a nonperturbative manner, regardless of near degeneracies or the number of open shells. As an application, the $\mathrm{Li}$ ground $\left({ }^{2} S\right)$ and first excited ${ }^{2} P^{\circ}$ states were studied. The results are collected in Tables $I$ and $I I$ and in Figs. 1-3. These values would change only by a small percentage if completely correlated wave functions were used, but, of course, their meanings would not.
[1] L. R. Hunter, D. Krause, Jr., D. J. Berkeland, and M. G. Boshier, Phys. Rev. A 44, 6140 (1991).

[2] R. W. Molof, H. Schartz, T. Miller, and B. Bederson, Phys. Rev. A 10, 1131 (1974).

[3] J. S. Sims, S. A. Hagstrom, and J. R. Rumble, Jr., Phys. Rev. A 14, 576 (1976).

[4] R. W. Schmieder, A. Lurio, and W. Happer, Phys. Rev. A 3, 1209 (1971).

[5] S. A. Adelman and A. Szabo, J. Chem. Phys. 58, 687 (1973).

[6] C. A. Nicolaides and S. I. Themelis, Phys. Rev. A 45, 349 (1992).

[7] C. A. Nicolaides, Th. Mercouris, and G. Aspromallis, J. Opt. Soc. Am. B 7, 494 (1990).

[8] C. A. Nicolaides, Th. Mercouris, and N. A. Piangos, J. Phys. B 23, L669 (1990).
[9] C. A. Nicolaides and Th. Mercouris, Phys. Rev. A 44, 7827 (1991).

[10] V. A. Davydkin and V. D. Ovsiannikov, J. Phys. B 19, 2071 (1986).

[11] C. A. Nicolaides, Phys. Rev. A 6, 2078 (1972); C. A. Nicolaides and D. R. Beck, Int. J. Quantum Chem. 14, 457 (1978).

[12] R. M. Glover and F. Weinhold, J. Chem. Phys. 65, 4913 (1976).

[13] Field-induced polarization terms are important in the final-state core terms for negative-ion dc field resonances, as it was shown from the computations on $\mathrm{Li}^{-}$of $\mathrm{C}$. A. Nicolaides and Th. Mercouris, Chem. Phys. Lett. 159, 45 (1989) (Fig. 2).

[14] I. I. Sobel'man, Introduction to the Theory of Atomic Spectra (Pergamon, New York, 1972), Chap. 8. 